\begin{tabular}{|c|c|c|c|}
\hline REs & $\begin{array}{l}\text { Cloning } \\
\text { Sites }\end{array}$ & Oligos/Primers (5' to 3') & Reference \\
\hline $\begin{array}{l}\text { MAPK/ERK } \\
\text {-RE }\end{array}$ & $\begin{array}{l}\text { BstBI, } \\
\text { HpaI }\end{array}$ & $\begin{array}{l}\text { For: } \\
\text { AACGGATGTCCATATTAGGAGGATGTCCATATTAGGAGGATGTCCATATT } \\
\text { AGGAGGATGTCCATATTAGGAGGATGTCCATATTAGGATT } \\
\text { Rev: } \\
\text { CGAATCCTAATATGGACATCCCCCTAATATGGACATCCTCCTAATATGGA } \\
\text { CATCCTCCTAATATGGACATCCTCCTAATATGGACATCCGTT }\end{array}$ & SABiosciences, inc. \\
\hline CArG-RE & $\begin{array}{l}\text { BstBI, } \\
\text { HpaI }\end{array}$ & $\begin{array}{l}\text { For: } \\
\text { AACCCATAAAAGGCCATAAAAGGCCATAAAAGGCCATAAAAGGCCATAA } \\
\text { AAGGCCATAAAAGGTT } \\
\text { Rev: } \\
\text { CGAACCTTTTATGGCCTTTTATGGCCTTTTATGGCCTTTTATGGCCTTTTAT } \\
\text { GGCCTTTTATGGGTT }\end{array}$ & SABiosciences, inc. \\
\hline CArGA-RE & $\begin{array}{l}\text { BstBI, } \\
\text { HpaI }\end{array}$ & $\begin{array}{l}\text { For: } \\
\text { AACCCTTGTTTGGCCTTGTTTGGCCTTGTTTGGCCTTGTTTGGCCTTGTTTG } \\
\text { GCCTTGTTTGGTT } \\
\text { Rev: } \\
\text { CGAACCAAACAAGGCCAAACAAGGCCAAACAAGGCCAAACAAGGCCAA } \\
\text { ACAAGGCCAAACAAGGGTT }\end{array}$ & (1) \\
\hline EGR1-RE & $\begin{array}{l}\text { BstBI, } \\
\text { HpaI }\end{array}$ & $\begin{array}{l}\text { For: } \\
\text { AACCGCCCCCGCGCGCCCCCGCGCGCCCCCGCGCGCCCCCGCGCGCCCCC } \\
\text { GCGCGCCCCCGCGTT } \\
\text { Rev: } \\
\text { CGAACGCGGGGGCGCGCGGGGGCGCGCGGGGGCGCGCGGGGGCGCGCG } \\
\text { GGGGCGCGCGGGGGCGGTT }\end{array}$ & SABiosciences, inc. \\
\hline KLF4-RE & $\begin{array}{l}\text { BstBI, } \\
\text { HpaI }\end{array}$ & $\begin{array}{l}\text { For: } \\
\text { AACAGGGTGTGGCCAGGGTGTGGCCAGGGTGTGGCCAGGGTGTGGCCAG } \\
\text { GGTGTGGCCAGGGTGGGCCTT } \\
\text { Rev: } \\
\text { CGAAGGCCACACCCTGGCCACACCCTGGCCACACCCTGGCCACACCCTGG } \\
\text { CCACACCCTGGCCACACCCTGTT }\end{array}$ & SABiosciences, inc. \\
\hline MEF2-RE & $\begin{array}{l}\text { BstBI, } \\
\text { BsiWI }\end{array}$ & $\begin{array}{l}\text { For: } \\
\text { GTACGCTAGCGCTCTAAAAATAACCCTCTAGCGCTCTAAAAATAACCCTC } \\
\text { TAGCGCTCTAAAAATAACCCTCTAGCGCTCTAAAAATAACCCTTTT } \\
\text { Rev: } \\
\text { CGAAAGGGTTATTTTTAGAGCGCTAGAGGGTTATTTTTAGAGCGCTAGAG } \\
\text { GGTTATTTTTAGAGCGCTAGAGGGTTATTTTAAGAGCGCTAGC }\end{array}$ & (2) \\
\hline SP1-RE & $\begin{array}{l}\text { BstBI, } \\
\text { BsiWI }\end{array}$ & $\begin{array}{l}\text { For: } \\
\text { GTACGGGGGCGGGGCGGGGCGGGGCGGGGCGGGGCGGGGCGGGGCGGG } \\
\text { GCGGGGCGGGGCGGGCTT } \\
\text { Rev: } \\
\text { CGAAGCCCCGCCCCGCCCCGCCCCGCCCCGCCCCGCCCCGCCCCGCCCCG } \\
\text { CCCCGCCCCGCCCCC }\end{array}$ & SABiosciences, inc. \\
\hline ATF6-RE & $\begin{array}{l}\text { BstBI, } \\
\text { BsiWI }\end{array}$ & $\begin{array}{l}\text { For: } \\
\text { GTACGCTCGAGACAGGTGCTGACGTGGCATTCCTCGAGACAGGTGCTGAC } \\
\text { GTGGCATTCCTCGAGACAGGTGCTGACGTGGCATTCTT } \\
\text { Rev: } \\
\text { CGAAGAATGCCACGTCAGCACCTGTCTCGAGGAATGCCACGTCAGCACCT } \\
\text { GTCTCGAGGAATGCCACGTCAGCACCTGTCTCGAGC }\end{array}$ & SABiosciences, inc. \\
\hline
\end{tabular}




\begin{tabular}{|c|c|c|c|}
\hline HIF1-RE & $\begin{array}{l}\text { BstBI, } \\
\text { BsiWI }\end{array}$ & $\begin{array}{l}\text { For: } \\
\text { GTACGTACGTGCTTACGTGCTTACGTGCTTACGTGCTTACGTGCTTACGTG } \\
\text { CTTT } \\
\text { Rev: } \\
\text { CGAAAGCACGTAAGCACGTAAGCACGTAAGCACGTAAGCACGTAAGCAC } \\
\text { GTAC }\end{array}$ & SABiosciences, inc. \\
\hline Nanog-RE & $\begin{array}{l}\text { BstBI, } \\
\text { BsiWI }\end{array}$ & $\begin{array}{l}\text { For: } \\
\text { GTACGACCCTTCGCCGATTAAGTACTTAAACCCTTCGCCGATTAAGTACTT } \\
\text { AAACCCTTCGCCGATTAAGTACTTAATT } \\
\text { Rev: } \\
\text { CGAATTAAGTACTTAATCGGCGAAGGGTTTAAGTACTTAATCGGCGAAGG } \\
\text { GTTTAAGTACTTAATCGGCGAAGGGTC }\end{array}$ & SABiosciences, inc. \\
\hline STAT3-RE & $\begin{array}{l}\text { BstBI, } \\
\text { BsiWI }\end{array}$ & $\begin{array}{l}\text { For: } \\
\text { GTACGGTCGACATTTCCCGTAAATCGTCGAGTCGACATTTCCCGTAAATC } \\
\text { GTCGAGTCGACATTTCCCGTAAATCGTCGATT } \\
\text { Rev: } \\
\text { CGAATCGACGATTTACGGGAAATGTCGACTCGACGATTTACGGGAAATGT } \\
\text { CGACTCGACGATTTACGGGAAATGTCGACC }\end{array}$ & SABiosciences, inc. \\
\hline p53-RE & $\begin{array}{l}\text { BstBI, } \\
\text { BsiWI }\end{array}$ & $\begin{array}{l}\text { For: } \\
\text { GTACGAGACATGTCCAGACATGTCCGAACATGTCCCAACATGTTGTAGAC } \\
\text { ATGTCCAGACATGTCCGAACATGTCCCAACATGTTGTTT } \\
\text { Rev: } \\
\text { CGAAACAACATGTTGGGACATGTTCGGACATGTCTGGACATGTCTACAAC } \\
\text { ATGTTGGGACATGTTCGGACATGTCTGGACATGTCTC }\end{array}$ & SABiosciences, inc. \\
\hline $\begin{array}{l}\text { SMAD2/3- } \\
\text { RE }\end{array}$ & $\begin{array}{l}\text { BstBI, } \\
\text { BsiWI }\end{array}$ & $\begin{array}{l}\text { For: } \\
\text { GTACGAGCCAGACAAGCCAGACAAGCCAGACAAGCCAGACAAGCCAGA } \\
\text { CAAGCCAGACATT } \\
\text { Rev: } \\
\text { CGAATGTCTGGCTTGTCTGGCTTGTCTGGCTTGTCTGGCTTGTCTGGCTTG } \\
\text { TCTGGCTC }\end{array}$ & SABiosciences, inc. \\
\hline SMAD4-RE & $\begin{array}{l}\text { BstBI, } \\
\text { BsiWI }\end{array}$ & $\begin{array}{l}\text { For:GTACGGGCGCGGGCGCGGGCGCGGGCGCGGGCGCGGGCGCGGGCGC } \\
\text { GTT } \\
\text { Rev:CGAACGCGCCCGCGCCCGCGCCCGCGCCCGCGCCCGCGCCCGCGCCC }\end{array}$ & SABiosciences, inc \\
\hline SMAD7-RE & $\begin{array}{l}\text { BstBI, } \\
\text { BsiWI }\end{array}$ & $\begin{array}{l}\text { For: } \\
\text { GTACGTAGCCAGACAGTAGCCAGACAGTAGCCAGACAGTAGCCAGACAG } \\
\text { TAGCCAGACAGTAGCCAGACAGTAGCCAGACAGTT } \\
\text { Rev: } \\
\text { CGAACTGTCTGGCTACTGTCTGGCTACTGTCTGGCTACTGTCTGGCTACTG } \\
\text { TCTGGCTACTGTCTGGCTACTGTCTGGCTAC }\end{array}$ & (3) \\
\hline $\begin{array}{l}\text { SMAD1/5/8- } \\
\text { RE }\end{array}$ & $\begin{array}{l}\text { BstBI, } \\
\text { BsiWI }\end{array}$ & $\begin{array}{l}\text { For: FOR: CACAGTTAACGAGCTAACATAACCCGGGAGGT } \\
\text { Rev: CACATTCGAAACCAACAGTACCGGAATGCCAAGC }\end{array}$ & $\begin{array}{l}\text { Provided by Dr. } \\
\text { Blobe (4) }\end{array}$ \\
\hline Notch-RE & $\begin{array}{l}\text { BstBI, } \\
\text { HpaI }\end{array}$ & $\begin{array}{l}\text { For: } \\
\text { AACCGTGGGAACGTGGGAACGTGGGAACGTGGGAACGTGGGAACGTGGG } \\
\text { AATT } \\
\text { Rev: } \\
\text { CGAATTCCCACGTTCCCACGTTCCCACGTTCCCACGTTCCCACGTTCCCAC } \\
\text { GGTT }\end{array}$ & SABiosciences, inc. \\
\hline ACTA2-Pr & $\begin{array}{l}\text { EcoRI, } \\
\text { AgeI }\end{array}$ & $\begin{array}{l}\text { For: ACAACAGGATCCCTTCCTGTCAAGGAGGTTAG } \\
\text { Rev: ACAACAACCGGTCGGGTAATTAAAAGAGCCAC }\end{array}$ & (5) \\
\hline SM22-Pr & $\begin{array}{l}\text { EcoRI, } \\
\text { AgeI }\end{array}$ & $\begin{array}{l}\text { For: ACAACAGGATCCGGCCTGGTGTGGAGTAGGTC } \\
\text { Rev: ACAACAACCGGTGGTTCGGACAGACAGGATGG }\end{array}$ & (6) \\
\hline SMTNB-Pr & $\begin{array}{l}\text { EcoRI, } \\
\text { AgeI }\end{array}$ & $\begin{array}{l}\text { For: ACAACAGGATCCGGCCTCTGGCACTGAAGATG } \\
\text { Rev: ACAACAACCGGTGGATTCTGCGGAGAGCTGCC }\end{array}$ & (7) \\
\hline MKL1-Pr & ClaI, AgeI & $\begin{array}{l}\text { For: ACAACAATCGATCGGGCAAGGGTATAGGATTT } \\
\text { Rev: ACAACAACCGGTAACCAAGAGGGTGGGAAAGA }\end{array}$ & $\begin{array}{l}\text { http://www.switchdb } \\
\text {.com/ }\end{array}$ \\
\hline
\end{tabular}




\begin{tabular}{|c|c|c|c|}
\hline PITX2-Pr & ClaI, AgeI & $\begin{array}{l}\text { For: ACAACAATCGATCGTGATCTGAGGCGACTTTT } \\
\text { Rev: ACAACAACCGGTAAGAGTGACAGGGACAGGTA }\end{array}$ & (8) \\
\hline DES-Pr & $\begin{array}{l}\text { EcoRI, } \\
\text { ClaI }\end{array}$ & $\begin{array}{l}\text { For: ACAACAGAATTCTTTATGGGGTCAGGTAGTTG } \\
\text { Rev: ACAACAATCGATGTTGAGAGATTCAATTCTGC }\end{array}$ & $\begin{array}{l}\text { http://www.switchdb } \\
\text {.com/ }\end{array}$ \\
\hline CSRP2-Pr & ClaI, AgeI & $\begin{array}{l}\text { For: ACAACAATCGATGAGGTCATGAGGAGGCCTAG } \\
\text { Rev: ACAACAACCGGTCCTACTCACTTGAGTCGGAG }\end{array}$ & $\begin{array}{l}\text { http://www.switchdb } \\
\text {.com/ }\end{array}$ \\
\hline MKL2-Pr & ClaI, AgeI & $\begin{array}{l}\text { For: ACAACAATCGATTTGGGAAGTTTGTAAAGTGA } \\
\text { Rev: ACAACAACCGGTGACGAACAGGGATGGCTGCC }\end{array}$ & $\begin{array}{l}\text { http://www.switchdb } \\
\text {.com/ }\end{array}$ \\
\hline ACTB-Pr & ClaI, AgeI & $\begin{array}{l}\text { For: ACAACAATCGATATAGCAGACATACAACGGAC } \\
\text { Rev: ACAACAACCGGTCAAAGGCGAGGCTCTGTGCT }\end{array}$ & $\begin{array}{l}\text { http://www.switchdb } \\
\text {.com/ }\end{array}$ \\
\hline $\begin{array}{l}\text { rMYH11-Pr } \\
\text { (rabbit) }\end{array}$ & ClaI, AgeI & $\begin{array}{l}\text { For: ACAACAATCGATTCCACACTCAGCCATCTCAC } \\
\text { Rev: ACAACAACCGGTAGCAAGAGCTCCACAACTCA }\end{array}$ & (9) \\
\hline
\end{tabular}

\section{Supplemental References:}

1. Hautmann MB, Madsen CS, Owens GK. A transforming growth factor beta (TGFbeta) control element drives TGFbeta-induced stimulation of smooth muscle alpha-actin gene expression in concert with two CArG elements. J Biol Chem. 1997;272(16):10948-56.

2. Lu J, McKinsey TA, Nicol RL, Olson EN. Signal-dependent activation of the MEF2 transcription factor by dissociation from histone deacetylases. Proc Natl Acad Sci U S A. 2000;97(8):4070-5.

3. Bajpai VK, Mistriotis P, Loh YH, Daley GQ, Andreadis ST. Functional vascular smooth muscle cells derived from human induced pluripotent stem cells via mesenchymal stem cell intermediates.

Cardiovasc Res. 2012;96(3):391-400.

4. Bajpai VK, Mistriotis P, Andreadis ST. Clonal multipotency and effect of long-term in vitro expansion on differentiation potential of human hair follicle derived mesenchymal stem cells. Stem Cell Res. 2012;8(1):74-84.

5. Reddy S, Ozgur K, Lu M, Chang W, Mohan SR, Kumar CC, et al. Structure of the human smooth muscle alpha-actin gene. Analysis of a cDNA and 5' upstream region. J Biol Chem. 1990;265(3):1683-7.

6. Solway J, Seltzer J, Samaha FF, Kim S, Alger LE, Niu Q, et al. Structure and expression of a smooth muscle cell-specific gene, SM22 alpha. J Biol Chem. 1995;270(22):13460-9.

7. Rensen SS, Thijssen VL, De Vries CJ, Doevendans PA, Detera-Wadleigh SD, Van Eys GJ. Expression of the smoothelin gene is mediated by alternative promoters. Cardiovasc Res. 2002;55(4):850-63.

8. Kioussi C, Briata P, Baek SH, Rose DW, Hamblet NS, Herman T, et al. Identification of a Wnt/Dvl/beta-Catenin --> Pitx2 pathway mediating cell-type-specific proliferation during development. Cell. 2002;111(5):673-85.

9. Katoh Y, Loukianov E, Kopras E, Zilberman A, Periasamy M. Identification of functional promoter elements in the rabbit smooth muscle myosin heavy chain gene. J Biol Chem. 1994;269(48):30538-45. 